\title{
A new ball launching system with controlled flight parameters for catching experiments
}

\author{
A. d'Avella ${ }^{\mathrm{a}, *}$, B. Cesqui a ${ }^{\text {, A. Portone }}{ }^{\mathrm{a}, \mathrm{b}}$, F. Lacquaniti ${ }^{\mathrm{a}, \mathrm{b}, \mathrm{c}}$ \\ a Laboratory of Neuromotor Physiology, Santa Lucia Foundation, via Ardeatina 306, 00179 Rome, Italy \\ b Department of Neuroscience, University of Rome, "Tor Vergata", via Montpellier 1, 00133 Rome, Italy \\ ' Center of Space Biomedicine, University of Rome, “Tor Vergata”, via Raimondo 18, 00173 Rome, Italy
}

\section{A R T I C L E I N F O}

\section{Article history:}

Received 21 October 2010

Received in revised form 11 January 2011

Accepted 12 January 2011

\section{Keywords:}

Actuated apparatus

Interception

Automatic control

Air drag

Polynomial fit

Ball flight parameters

\begin{abstract}
A B S T R A C T
Systematic investigations of sensorimotor control of interceptive actions in naturalistic conditions, such as catching or hitting a ball moving in three-dimensional space, requires precise control of the projectile flight parameters and of the associated visual stimuli. Such control is challenging when air drag cannot be neglected because the mapping of launch parameters into flight parameters cannot be computed analytically. We designed, calibrated, and experimentally validated an actuated launching apparatus that can control the average spatial position and flight duration of a ball at a given distance from a fixed launch location. The apparatus was constructed by mounting a ball launching machine with adjustable delivery speed on an actuated structure capable of changing the spatial orientation of the launch axis while projecting balls through a hole in a screen hiding the apparatus. The calibration procedure relied on tracking the balls with a motion capture system and on approximating the mapping of launch parameters into flight parameters by means of polynomials functions. Polynomials were also used to estimate the variability of the flight parameters. The coefficients of these polynomials were obtained using the launch and flight parameters of 660 launches with 65 different initial conditions. The relative accuracy and precision of the apparatus were larger than $98 \%$ for flight times and larger than $96 \%$ for ball heights at a distance of $6 \mathrm{~m}$ from the screen. Such novel apparatus, by reliably and automatically controlling desired ball flight characteristics without neglecting air drag, allows for a systematic investigation of naturalistic interceptive tasks.
\end{abstract}

(C) 2011 Elsevier B.V. All rights reserved.

\section{Introduction}

The identification of which mechanisms underlie our use of visual information and prior knowledge of physical laws to guide interceptive actions is still an open question (Zago et al., 2009). Intercepting a moving object is a demanding task because visual stimuli guiding movement execution change in time and the central nervous system (CNS) must update on-line or predict the object position to compensate for sensorimotor latencies and bring the arm to the right place at the right time. To date, several experimental paradigms have been used to investigate the sensorimotor control mechanisms employed by the CNS to accomplish a variety of interceptive tasks. Motor responses have been characterized when capturing or punching an object moving along a linear path such as a ball dropped vertically with different motions (Lacquaniti

\footnotetext{
* Corresponding author. Tel.: +3906 51501484; fax: +39 0651501482 E-mail addresses: a.davella@hsantalucia.it (A. d'Avella), b.cesqui@hsantalucia.it (B. Cesqui), a.portone@hsantalucia.it (A. Portone), lacquaniti@caspur.it (F. Lacquaniti).
}

et al., 1993; Lacquaniti and Maioli, 1989, 1987; Zago et al., 2005; Zago and Lacquaniti, 2005), when catching (Bennett et al., 1999) or striking (Tresilian and Lonergan, 2002; Tresilian and Plooy, 2006; Tresilian et al., 2009) a target moving along a horizontal linear track, when intercepting targets moving on a computer monitor (Brenner and Smeets, 2007; Brouwer et al., 2000, 2002a,b, 2003; Mrotek and Soechting, 2007) or on a head-mounted display (Senot et al., 2005), when intercepting a ball attached on a rod and dropped from the ceiling of the room (Dessing et al., 2009; Peper et al., 1994), and when catching, hitting or batting a ball thrown by a launching machine, both while standing still (Brouwer et al., 2002b; Button et al., 2002; Land and McLeod, 2000; Laurent et al., 1994; Mazyn et al., 2006, 2007; Montagne et al., 1999; Regan, 1997; Savelsbergh et al., 2002; Tijtgat et al., 2009) and while running to reach the ball (McBeath et al., 1995; McLeod and Dlenes, 1993; McLeod et al., 2001).

In particular, tasks requiring the interception of balls flying in three-dimensional space offer a powerful tool to investigate the motor control strategies exploited by humans to accomplish interceptive actions. Indeed, they require the correct interpretation of motion in depth (Regan et al., 1979; Regan and Beverley, 1978) 
and of the retinal expansion of the object image while approaching a subject (Lee, 1980). Motor responses are influenced by the kinematic characteristics of the ball, estimated from retinal images (Zago et al., 2009), and from other visual cues providing additional information on the position and velocity during motion as well as other intrinsic characteristics of the ball such as mass, dimensions, and geometry (Lopez-Moliner et al., 2007a,b; Savelsbergh et al., 2002; Shepard, 1994). Moreover, the prediction of the ball trajectory, necessary for fast moving objects, requires knowledge of dynamic behavior of the flying projectile (Shepard, 1994; Tresilian, 1999a,b; van der Kamp et al., 1997; Zago et al., 2009), such as the effect that the gravitational field exerts on the trajectory. The integration of all these sources of information allows the CNS to predict the spatial and temporal evolution of the ball trajectory in space, and to trigger the appropriate motor response to accomplish the task.

However, while catching flying balls in three-dimensional space is an effective paradigm to study sensorimotor coordination, it is generally difficult to control the ball flight parameters. Indeed, the effects that air drag exerts on a flying object induce mathematical complications in the formulation of the equations describing its motion, and in the case of non-linear drag there are no possible analytical solutions (De Mestre, 1990). In previous studies balls were projected by means of commercial launching machines used to train athletes from different sport disciplines such as cricket and tennis (Bockemuhl et al., 2009; Button et al., 2002; Laurent et al., 1994; Mazyn et al., 2006, 2007; Tijtgat et al., 2009; Wann, 1996). In many cases, the experimenter manually positioned the launcher, by trial and error over many test launches, with a specific orientation and manually set the rotational speed of its delivery wheels to ensure desired interception height and flight time at a given distance from the launcher. Such methodology has some limitations. First, it does not ensure standardized visual stimuli at launch. Different flight parameters require different spatial orientations of the launcher and thus different positions of the ball when it is released, unless the pivot of the launching machine is aligned with the delivery hole. In all other cases, as with most commercial launching machines, the position of the launcher must be adjusted as a function of its orientation in order to ensure a fixed ball position at launch time. Second, it does not permit easily randomized sequences of launches. One may want to design an experiment with unpredictable ball trajectories in which participants are asked to catch balls projected each time with different flight parameters. To do so, it should be possible to change the configuration of the launching apparatus quickly and reliably, thus speeding up the duration of the experimental session. Third, it does not prevent visual anticipation due to the visibility of the ball prior to its release, possibly affecting the estimation of response timing. Finally, it does not allow quantification of random flight variability. Non-homogeneous ball surface, possible vibration of the apparatus, and accidental application of initial velocity to the ball during the insertion phase are some of the possible factors unpredictably altering ball launch parameters. Also, the lighter the ball (a desirable feature for safety), the stronger the air drag affects ball motion during flight. Thus, as a certain amount of variability cannot be avoided, it is crucial to monitor the ball trajectory to fully characterize the relationship between motor responses and ball kinematics.

Here we introduce a novel actuated launching system with an automatic launch parameter control that addresses some of the limitations of launching apparatus used previously to investigate catching in three-dimensional space. The system comprises a commercial launching machine and a custom-made supporting structure. The system is actuated and calibrated in order to guarantee the rapid positioning and orientation of the launcher and to ensure the achievement of the desired ball flight parameters without neglecting air drag. Furthermore, the ball trajectory is tracked by a motion capture system. We also present the procedure used to calibrate the apparatus based on a large number of ball trajectories and the results of two validation tests carried out to quantify the accuracy and precision achieved in controlling ball flight parameters.

\section{Materials and methods}

The launching system has been designed for projecting balls from a fixed initial location to a desired location on a vertical plane, at a given distance from the initial location, and with a desired flight duration. The appropriate ball trajectories were generated by adjusting the initial ball velocity vector, e.g. initial speed (SP), elevation angle (EL), and azimuth angle (AZ) taking into account air drag. The functional relationship between launch and flight parameters was estimated through a calibration procedure based on a large set of recorded ball trajectories. To allow for rapid change and repeatability of the desired flight parameters, the launch parameters (SP, EL, AZ) were set automatically by computer controlled actuators.

\subsection{Launching apparatus}

The launching apparatus was constructed by mounting a commercial ball launching machine, used to train crickets players, with a fixed launch axis and an adjustable ball exit speed, on a custommade articulated and actuated structure that could displace and orient the launching machine (Fig. 1). The whole structure was hidden by a large screen with a small hole from which the ball was projected (Fig. 1A). The apparatus also include a motion capture system to track the ball trajectory.

\subsubsection{Launching machine}

The launching machine (Bola Professional Cricket Bowling Machine, Stewart and Williams, Bristol, UK) had two molded urethane ball delivery wheels, with concave profiles, rotating in opposite directions around two parallel axes that could deliver balls of approximately $7 \mathrm{~cm}$ in diameter along a fixed axis (launch axis) at speeds ranging from 15 to $95 \mathrm{mph}$ with a resolution of $1 \mathrm{mph}$. The machine was custom modified by the manufacturer to allow setting the launch speed remotely via an RS232 computer interface.

\subsubsection{Positioning structure}

The actuated structure used for positioning the launching machine was made of modular aluminum structural framing elements (Bosch Rexroth AG, Stuttgart Germany, assembled by RTC-Romana Trasmissioni Colme, s.r.l., Ariccia, Italy). The structure was composed by a supporting fixed outer frame and three moving inner frames (Fig. 1B). One side of the outer frame was covered with a large screen $(4 \mathrm{~m} \times 3 \mathrm{~m}$, width $\times$ height, foldable for storage) with a hole of $0.14 \mathrm{~m}$ of diameter and center at a height $\left(Z_{\text {exit }}\right)$ of $1.66 \mathrm{~m}$ from which the balls were projected (launch location).

The height of the hole could be easily changed by substituting the central panel of the screen. The three inner frames were interconnected through prismatic and rotational joints to provide four degrees-of-freedom to the launching machine: horizontal translation ( $Y$, where $X$ is the horizontal axis perpendicular to the screen), vertical translation $(Z)$, elevation rotation (EL, the angle between the launch axis and the horizontal plane) and azimuth rotation ( $\mathrm{AZ}$, the angle between the launch axis and the vertical plane containing the $X$ axis). The two translational degrees-of-freedom were required to allow the passage of the ball through the hole with the launching machine at different elevation and azimuth angles. The launching machine was mounted on the innermost frame (frame 1 , Fig. 1C) which was connected to the next frame (frame 2) through rotational bearings forming a rotational joint around a horizontal 
A

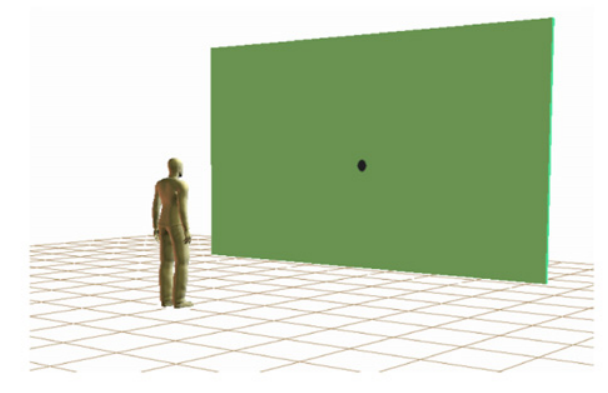

C

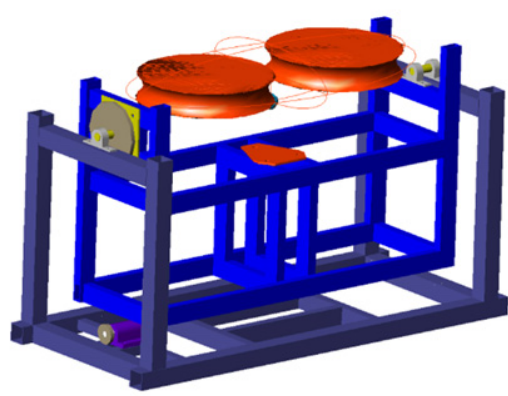

B
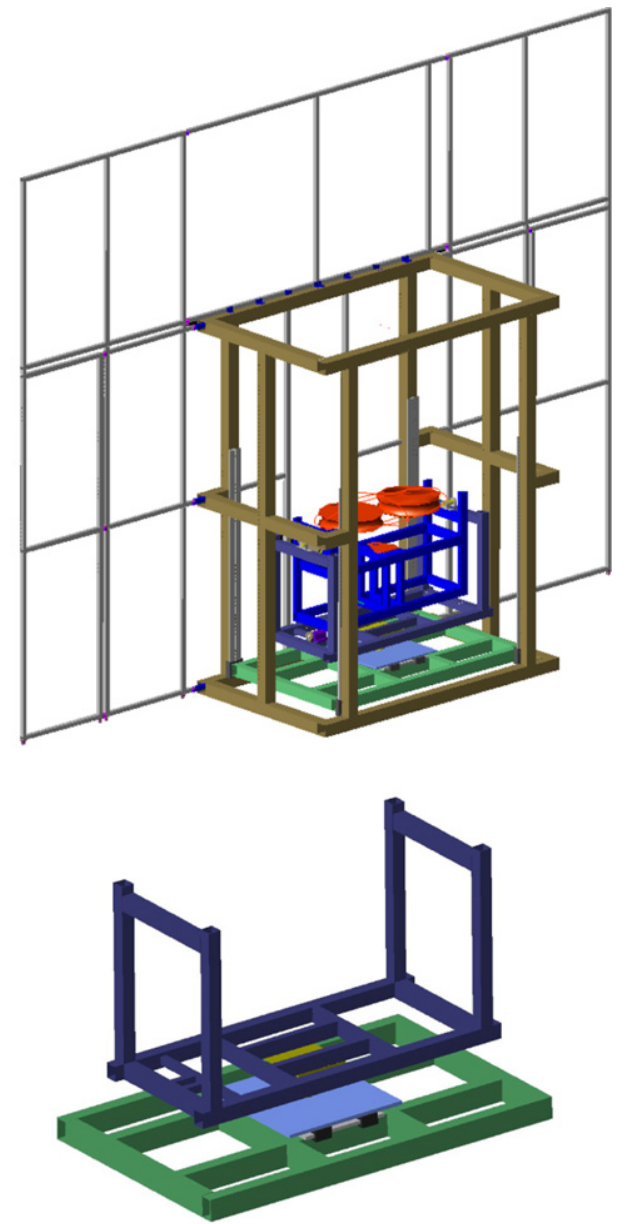

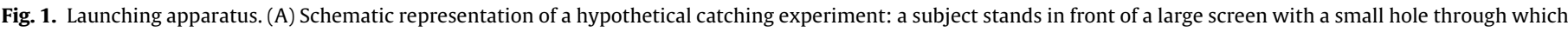

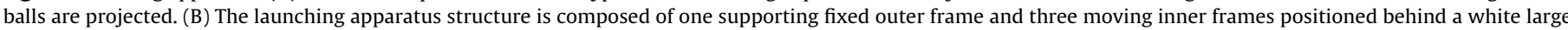

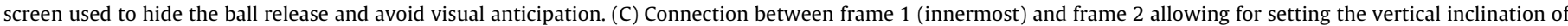

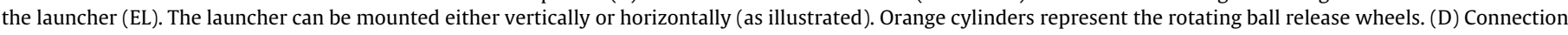

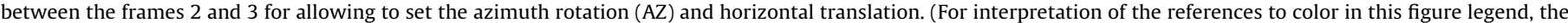
reader is referred to the web version of the article.)

axis that allowed to set the elevation angle of the machine launch axis. The launching machine could be mounted with the delivery wheels in either a vertical plane or in the horizontal plane (as illustrated in Fig. 1B). Actuation was provided by a sprocket-chain system powered by a stepper motor (SM 2863-5255, Sanyo Denki, America Inc. driven by the circuit SAC26, RTA, Marcignago, Pavia, Italy). Frame 2 was connected to frame 3 through a rotational and a prismatic joint (Fig. 1D). The rotational joint around the vertical axis allowed to various the azimuth angles. The prismatic joint along the horizontal axis $(Y)$ allowed translation of the machine to both sides of the exit hole. Both joints were actuated through a lead screw (OSP-E, Origa Hoerbiger, Zug, Switzerland), moved by two additional stepper motors (SM 2863-5255 Sanyo Denki, SAC26 driver circuit), acting, respectively, on a moving support rotating around a universal joint actuated with a sprocket-track system. Finally, frame 3 translated vertically with respect to the outer frame (Fig. 1B) through four vertical screws powered by a sprocket-chain system connected to a fourth stepper motor (SM 2863-5155, Sanyo, driven by an Xmind-B4 drive circuit, RTA, Marcignago, PV, Italy).

A set of geometrical parameters (see Table 1 for the definition of the symbols used throughout the text), necessary to coordinate the actuation of the structure different degrees-of-freedom (Fig. 2), were measured when the apparatus was installed. Specifically, the distance of the elevation rotation axis from the screen $\left(d_{\mathrm{a}}\right)$ was
$522 \mathrm{~mm}$, the distance between of the elevation rotation axis and the launch axis $\left(d_{\text {launch }}\right) 85 \mathrm{~mm}$.

\subsubsection{Control}

The controller of the automated structure was implemented on a standard personal computer. The four stepper motors were controlled by a dedicated PCI board (PCI-7330, National Instruments, Austin, TX, USA), which sent commands to the driver circuits through a dedicated interface (UMI 7764, National Instruments, Austin, TX, USA). The control software was developed in Labview 7.1 using Motion Control Tools (National Instruments, Austin, TX, USA) and allowed to control over the 4 degrees-of-freedom (or motion axis) independently during the calibration maneuvers (see Section 2.2) and simultaneously during the experimental sessions. In general, the relation between the number of steps of the motor (n) from a known motion axis reference configuration, at which the stepper motor counter is set to zero, and the translation or rotation of the launching machine $(A)$ with respect to the position or rotation $(C)$ at the reference configuration, is:

$A=K \times n+C$,

where $K$ is a known parameter characteristic of the transmission train of the specific motion axis. A desired configuration of the positioning structure is then achieved by sending a number of step advancement control pulses (according to Eq. (1)) to the stepper 
Table 1

Tables of symbols of variables and parameters used in the text.

\begin{tabular}{|c|c|}
\hline Symbol & Definition \\
\hline $\boldsymbol{a}_{\text {hole }}$ & Horizontal axis passing through the center of the hole on the screen and perpendicular to the screen \\
\hline $\boldsymbol{a}_{\text {launch }}$ & Launch axis, directed as the ball velocity at release from the delivery wheels \\
\hline $\boldsymbol{a}_{\text {laser }}$ & Laser beam axis \\
\hline$d_{\text {bola }}$ & Distance between the screen and the position of the ball at release \\
\hline$d_{\mathrm{a}}$ & Distance between elevation axis and screen plane \\
\hline$d_{\text {launch }}$ & Distance between of the elevation rotation axis and the launch axis \\
\hline$Z_{\text {exit }}$ & Vertical coordinate of the screen hole \\
\hline$Z_{\text {launcher }}$ & Vertical coordinate of the elevation axis \\
\hline $\mathrm{v}_{i}$ & Ball velocity at release from the delivery wheels \\
\hline$v_{0}$ & Ball velocity at the launch hole \\
\hline EL & Vertical inclination of the launcher \\
\hline $\mathrm{EL}_{\text {offset }}$ & Difference between the vertical inclination of the laser beam axis and the launch axis \\
\hline $\mathrm{EL}_{0}$ & Vertical inclination of the velocity vector $v_{0}$ \\
\hline
\end{tabular}

motor of each motion axis. Thus, $C$ must be determined in advance for each motion axis with a calibration procedure described below (Section 2.2).

For brevity, only the calibration and launch parameter estimation procedures for the elevation angle and vertical translation will be described in the following sections. The procedures for the azimuth angle and horizontal translation are similar and, for the launch parameter estimation, simpler because they do not require taking into account the gravitational acceleration on the flight trajectory.

\subsubsection{Ball tracking}

The launching apparatus also included a motion capture system (9-TV cameras Vicon-612 system, Oxford, UK), to track the ball trajectory both for calibration and experimental data collection. A large tracking volume $(7.5 \mathrm{~m} \times 3 \mathrm{~m} \times 3 \mathrm{~m})$ was required to capture the ball motion up to a distance of $7.5 \mathrm{~m}$ and for different flight times and final heights. The reconstruction residual, averaged over the 9 cameras, obtained with the calibration procedure and software provided by the manufacturer was $1.2 \mathrm{~mm}$. Tracking of lightweight expanded polyurethane balls (diameter $7 \mathrm{~cm}$, weight $20 \mathrm{~g}$ ) was achieved by covering their surface with retro-reflective tape (Scotchlite, 3M, Pioltello, Milan. Italy), cutting out a flattened icosahedron from a sheet of adhesive tape (Fig. 3).
A photo-sensor (E3T-S112, Omron Electronics S.p.A., Milan, Italy) mounted on the exit hole detected the instant at which the ball passed through the screen (launch time).

\subsection{Alignment of the apparatus to the reference position}

The procedure consisted of aligning the launch axis of the Bola machine ( $\boldsymbol{a}_{\text {launch }}$ of Fig. 2, hence, the axis directed as the initial velocity vector of the ball as it leaves the delivery wheels) to the horizontal axis orthogonal to the screen plane and passing through the center of the exit hole ( $\boldsymbol{a}_{\text {hole }}$ axis of Fig. 2 ), and setting the stepper motor counter for the EL and $Z$ axes at zero in this configuration. Thus $C_{\mathrm{EL}}$ and $C_{Z}$ are then set to zero and $Z_{\text {exit }}$, respectively, in the control Eq. (1).

In order to align the launch axis to the hole axis, a laser beam device was mounted on the Bola machine frame. The laser device could be oriented manually and it was regulated in order to align approximately the laser beam parallel ( $\boldsymbol{a}_{\text {laser }}$ of Fig. 2 ) to the launch axis. Then, moving the apparatus ( $Z$ and EL motion axes), the laser beam was aligned to the hole axis. To this end, it was verified that the interception of the laser beam with vertical planes parallel to the screen at different distances $(1,3$, and $6 \mathrm{~m})$ remained constant (same $y$ and $z$ coordinates). The entire structure was then vertically translated downward to set the height of the center of the delivery wheels with the center of the hole, and the resulting configuration

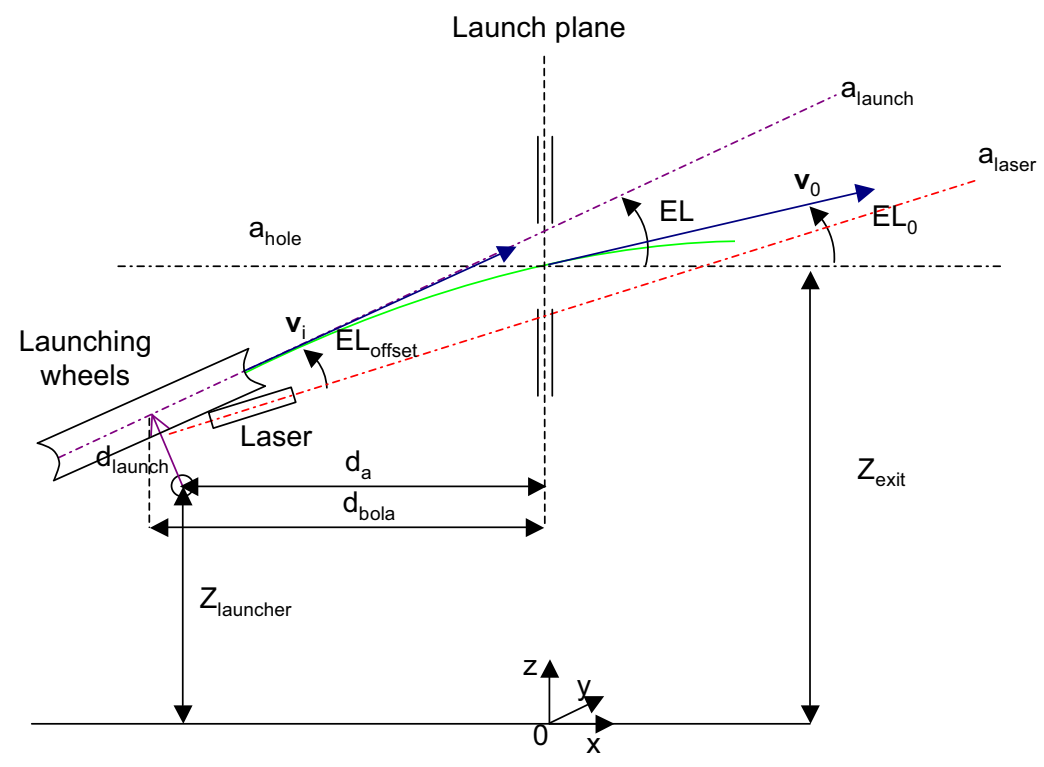

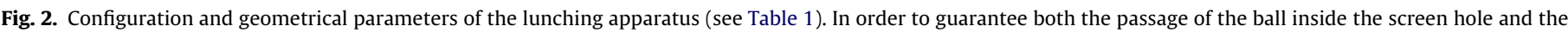

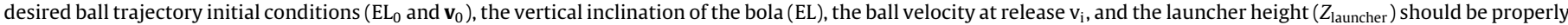
set. 

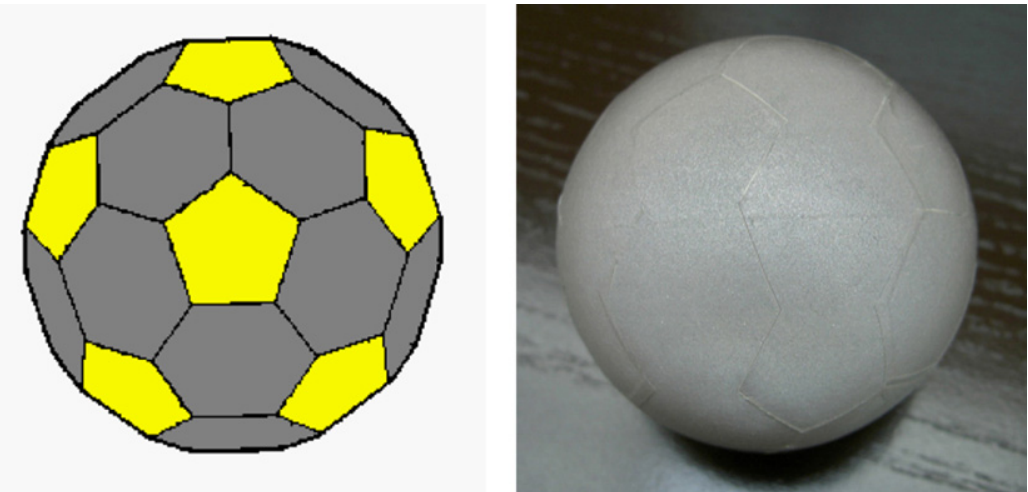

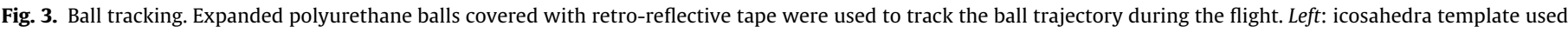
to apply the tape on the ball. Right: photograph of a ball used in the system.

of the apparatus was defined as the "laser reference configuration". However, since the manual adjustment of the laser device on the structure could not guarantee a precise alignment of the laser beam with the launch axis, it was necessary to estimate their small misalignment $\left(\mathrm{EL}_{\text {offset }}\right)$ with an experimental procedure. Such estimation was done by measuring the ball velocity at the exit hole and fitting the parameters of a simple model of the ball trajectory from the delivery wheels (i.e. the instant at which the ball moves along the launch axis) to the exit hole.

A set of launches, characterized by different combinations of EL and SP values were performed using the laser reference configuration to reset the $Z$ and EL stepper motor counters and to set $C_{\mathrm{EL}}$ and $C_{Z}$. Tracking, filtering and differentiating the ball spatial position was performed as further described in Section 2.3.2.1. The offset angle was obtained from the collected data by iterative optimization of an error function defined as:

$\operatorname{Err}=\sum_{k}\left\|\hat{\mathbf{v}}_{0 k}-\mathbf{v}_{0 k}\right\|^{2}$

where $\mathbf{v}_{0 k}$ is the velocity at the exit of the hole screen in the $k$ th launch trial, measured from real data collected with the motion tracking system, and $\hat{\mathbf{v}}_{0 k}$ is the velocity estimated according to the model:

$\left[\begin{array}{l}\hat{v}_{0 x} \\ \hat{v}_{o z}\end{array}\right]=\left[\begin{array}{l}f\left(\mathrm{EL}_{\text {offset }}, X_{\text {data }}\right) \\ g\left(\mathrm{EL}_{\text {offset }}, X_{\text {data }}\right)\end{array}\right]$

were, $X_{\text {data }}=(\mathrm{EL}, \mathrm{SP}, \mathrm{par})$, and the $f e g$ functions are given by the equations for the velocity of a projectile launched with initial velocity $\mathbf{v}_{i}$, with gravity acceleration $g$ and neglecting air drag:

$\left\{\begin{array}{l}v_{x}(t)=\mathrm{v}_{i x} \\ v_{z}(t)=\mathrm{v}_{i z}-g t\end{array}\right.$

At $t=t_{0}$ (when the ball passes through the hole in the screen), we have:

$\left\{\begin{array}{l}\hat{v}_{x}\left(t_{0}\right)=\mathrm{SP} \cos \left(\mathrm{EL}+\mathrm{EL}_{\mathrm{offset}}\right) \\ \hat{v}_{z}\left(t_{0}\right)=\mathrm{SP} \sin \left(\mathrm{EL}+\mathrm{EL}_{\text {offset }}\right)-g \frac{d_{\text {bola }}}{\mathrm{SP} \cos \left(\mathrm{EL}+\mathrm{EL}_{\text {offset }}\right)}\end{array}\right.$

where EL is the elevation angle set according to Eq. (1) with the laser reference configuration and

$d_{\text {bola }}=d_{\mathrm{a}}+d_{\text {launch }} \sin \left(\mathrm{EL}+\mathrm{EL}_{\text {offset }}\right)$

Once evaluated, $\mathrm{EL}_{\text {offset }}$ is used to define a new, more accurate reference configuration for the launching machine control software.

\subsection{Estimation of the launch parameters for desired flight parameters}

In practical applications, when using the launching system to vary systematically the experimental conditions, it is necessary to set the appropriate launch parameters EL and SP to obtain specific flight parameters, i.e. flight duration $T$, and ball height $Z$ at a distance $d$ from the launch plane. The problem can be divided in two parts. First, the velocity vector at the exit of the screen hole $\mathbf{v}_{0}$ generating the desired flight parameters $T$ and $Z$ at distance $d$ is estimated in real conditions, hence taking into account that the ball is affected by air drag. Second, the appropriate ball speed setting of the launcher (SP) and the appropriate position of the launcher, i.e. the elevation of its launch axis (EL) and the associated height from the ground $\left(Z_{\text {launcher }}\right)$ that guarantees ball passage through the hole of the screen with the required exit velocity $\mathbf{v}_{0}$ is determined.

\subsubsection{Definition of $Z_{\text {launcher }}$ apparatus configuration}

In order to permit the passage of the ball inside the hole of the screen, depending on the launch parameters EL and SP, the apparatus must be opportunely translated along the vertical axis. Thus, the controller has been programmed to sets the value of the launcher height $\left(Z_{\text {launcher }}\right)$ as a function of the launcher elevation (EL), according to

$Z_{\text {launcher }}=Z_{\text {exit }}-d_{\mathrm{a}} \tan (\mathrm{EL})$

relying on the simplifying assumptions that: (1) given the short distance from the delivery wheels and the exit hole, the effects of air drag and gravity on the ball trajectory can be neglected; (2) the distance $\left(d_{\text {launch }}\right)$ between launch axis ( $\left.\boldsymbol{a}_{\text {launch }}\right)$ and the elevation rotation axis, small compared to the distance $\left(d_{\mathrm{a}}\right)$ of the rotation axis from the screen, can also be neglected. These simplifying assumptions are reasonable because the approximations they introduce tend to cancel each other. Indeed, the first assumption (no drag, no gravity) tends to overestimate the height of the ball trajectory in the exit hole $\left(Z_{\text {exit }}\right)$, while the second tends to underestimate it.

\subsubsection{Relationship between launch and flight parameters}

Since $Z_{\text {launcher }}$ is determined once the inclination of the launcher is established with respect to the apparatus, it remains to define the relation between the desired flight parameters (flight time $T$ and trajectory height $Z$ at a distance $d$ from the screen) and the launch parameters (SP, EL). In addition, one may also want to control the velocity components in correspondence to the screen exit hole $\left(\mathbf{v}_{0}\right)$, hence acting on the initial visual stimuli available to a subject for interception of the ball. The original problem can be 


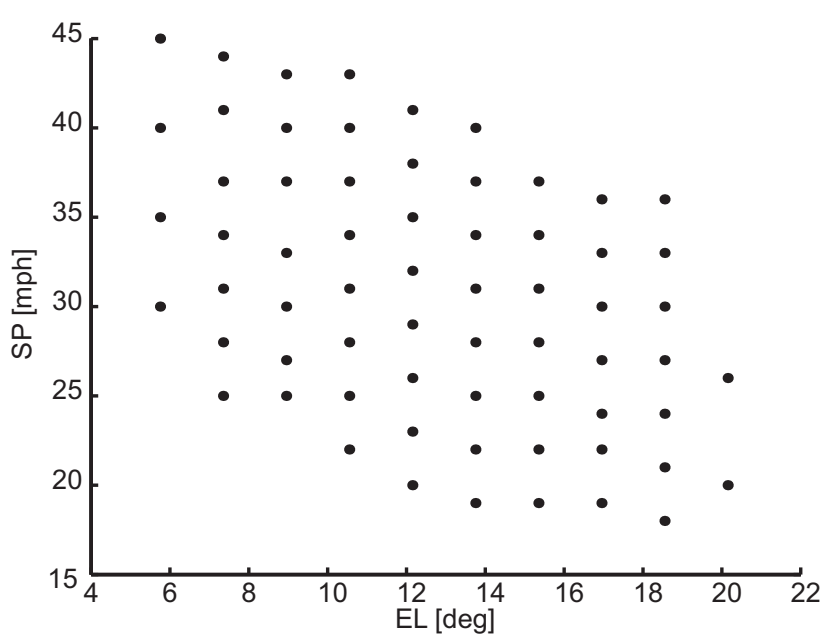

Fig. 4. Launch parameters used in the calibration experimental session. $X$-axis: elevation angle of the launcher $(\mathrm{EL})$ in degrees. $Y$-axis: ball speed at the time of release from the launcher delivery wheels (SP) in $\mathrm{mph}$.

then reformulated as identifying the following two mappings:

$$
(T, Z, d) \rightarrow\left(v_{0 x}, v_{0 z}\right) \rightarrow(\mathrm{EL}, \mathrm{SP})
$$

Taking into account the effects that air drag exerts on a flying object in the formulation of the equation describing its motion requires knowledge of several aerodynamic parameters which are not easily and reliably measurable. Moreover, for non-linear drag, the equations have no analytical solution and must be solved numerically (De Mestre, 1990). We thus adopted an empirical approach based on measured ball trajectories. We approximated the required mappings with polynomials of similar form to those that can be analytically derived from the motion equation neglecting air drag. In particular, we used the ball initial velocity and flight parameters from ball trajectories recorded during an experimental session to fit polynomials approximating the following mappings:

$$
\begin{aligned}
& v_{0 x}, v_{0 z} \rightarrow \hat{T}, \hat{Z} \\
& \mathrm{EL}, \mathrm{SP} \rightarrow \hat{v}_{0 x}, \hat{v}_{0 z} \\
& \mathrm{EL}, \mathrm{SP} \rightarrow \hat{\sigma}_{T}, \hat{\sigma}_{Z}
\end{aligned}
$$$$
\text { Flight parameters at distance } d \text { from the launch plane }
$$$$
\text { as a function of } v_{0}
$$$$
v_{0} \text {, velocity of the ball with respect to the exit hole of }
$$$$
\text { the screen as a function of lunch parameters }
$$
launch plane for each set of launch parameter values, EL and SP

2.3.2.1. Experimental procedure and data analysis (experiment 1). An experimental session of 660 launches was carried out setting 65 different pairs (one condition was repeated twice) of vertical inclinations (EL) and launching speeds (SP) as reported in Fig. 4. In particular EL and SP values were chosen in a range that was bounded by the height of the laboratory ceiling $(3.1 \mathrm{~m})$, to avoid any contact between the ball and the ceiling, and by the minimal flight duration appropriate for catching experiments. For each combination of EL and SP values a block of 10 launches were performed. The spatial position was tracked at $100 \mathrm{~Hz}$ using the motion capture system. Finally, ball motion coordinates were digitally low-passed filtered (FIR filter; $25 \mathrm{~Hz}$ cutoff frequency; Matlab filtfilt function), and the position and velocity at the launch plane and at vertical planes at specific distances $(d)$ from it were computed by fitting the ball trajectory (i.e. spatial coordinates as a function of time) around the positions of interest with a cubic splines (Matlab csaps function), differentiating them (Matlab fnder function), and evaluating them (Matlab fnval function) at the times of interception of the ball trajectory with the planes. As in a few launches (32/660) the motion capture system did not reconstruct the ball coordinates around the launch plane, missing samples were extrapolated using

second order polynomials. The same ball was used for the whole session.

2.3.2.2. $T$ and $Z$ estimation as a function of $v_{0 x}$ and $v_{0 z}$. If air drag is neglected, the relation between both $T$ and $Z$ at a given distance $d$ from the launch screen, with the initial velocity $\mathbf{v}_{0}$ can be computed considering the equation of the parabolic motion with respect to the coordinate frame of Fig. 2:

$\left\{\begin{array}{l}x(t)=v_{0 x} t \\ z(t)=z_{0}+v_{0 z} t-\frac{1}{2} g t^{2}\end{array}\right.$

where $v_{0 x}$ and $v_{0 z}$ are the velocity components and $z_{0}$ is the height of the ball with respect to of the exit hole of the screen (launch plane), $g$ is the acceleration due to gravity, directed along the $z$ axis. For a ball projected at $t=T, z(T)=Z$, and $x(T)=d, \mathbf{v}_{0}$ can be expressed as:

$$
\left\{\begin{array}{l}
v_{0 x}=\frac{d}{T} \\
v_{0 z}=\frac{Z-Z_{\text {exit }}}{T}+\frac{g T}{2}
\end{array}\right.
$$

Then:

$$
\left\{\begin{array}{l}
T=\frac{d}{v_{0 x}} \\
Z(T)=Z_{\text {exit }}+v_{0 z} T-\frac{g T^{2}}{2}=Z_{\text {exit }}+v_{0 z} \frac{d}{v_{0 x}}-\frac{g}{2}\left(\frac{d}{v_{0 x}}\right)^{2}
\end{array}\right.
$$

Multiplying the seconds equation by $v_{0 x}^{2}$ :

$Z(T) v_{0 x}^{2}=Z_{\text {exit }} v_{0 x}^{2}+v_{0 x} v_{0 z} d-\frac{g d}{2}$

Thus, according to Eq. (11), the height of ball arrival at distance $d$ multiplied by the square of the $x$ component of the initial ball velocity was approximated with a truncated third degree polynomial:

$$
\begin{aligned}
Z v_{0 x}^{2}= & a_{1} v_{0 x} v_{0 z} d+a_{2} v_{0 x}^{2}+a_{3} v_{0 z}^{2}+a_{4} d^{2}+a_{5} v_{0 x} v_{0 z}+a_{6} v_{0 x} d \\
& +a_{7} v_{0 z} d+a_{8} v_{0 x}+a_{9} v_{0 z}+a_{10} d+a_{11}
\end{aligned}
$$

Analogously, a second degree polynomial was used to express the relationship between $T$ and $\mathbf{v}_{0}$ :

$$
\begin{aligned}
T v_{0 x}= & b_{1} v_{0 x}^{2}+b_{2} v_{0 z}^{2}+b_{3} d^{2}+b_{4} v_{0 x} \mathrm{v}_{0 z}+b_{5} v_{0 x} d+b_{6} v_{0 z} d \\
& +b_{7} v_{0 x}+b_{8} v_{0 z}+b_{9} d+b_{10}
\end{aligned}
$$

The coefficients of the polynomial fits were estimated by least squares measuring the real flight time $T$, height $Z$ at a range of distances $d([4,4.5,5,5.5,6,6.5,7] \mathrm{m})$ from the screen plane, and $\mathbf{v}_{0}$ from the ball trajectory recorded during the experiment carried out for the calibration of the launching apparatus (see Section 2.3.2.1). The quality of the fit was evaluated by computing $R^{2}$ values computed as:

$R^{2}=1-\frac{\mathrm{SSE}}{\mathrm{SST}}$

where SSE is the sum of the squares of the differences the predicted values and the grand mean, and SST is the sum of the squares of the difference between the measured values and its grand mean.

2.3.2.3. $v_{0}$ estimation as a function of launch parameters. A similar approach has been used to approximate the mapping between the launch parameters (SP and EL) and the initial velocity $\mathbf{v}_{0}$. If we neglect air drag, the ball velocity at the launch plane, $v_{0 x}$ and $v_{0 z}$ 
can be expressed by means of analytical equations of a projectile launched with $v_{i x}$ and $v_{i z}$, initial velocity components:

$\left\{\begin{array}{l}v_{0 x}=v_{i x} \\ v_{0 z}=v_{i z}-g T\end{array}\right.$

If $T=d_{\text {bola }} / v_{i x}$, substituting we obtain:

$\left\{\begin{array}{l}v_{0 x}=v_{i x} \\ v_{0 z}=v_{i z}-\frac{g d_{\text {bola }}}{v_{i x}} \Rightarrow v_{0 z} v_{i x}=v_{i x} v_{i z}-g d_{\text {bola }}\end{array}\right.$

Thus, the dependence between $v_{0}$ and $v_{\mathrm{i}}$ can be approximated as follows:

$v_{0 x}=c_{1} v_{i x}+c_{2}$

$v_{i x} v_{0 z}=d_{1} v_{i x} v_{i z}+d_{2} v_{i x}^{2}+d_{3} v_{i z}^{2}+d_{4} v_{i x}+d_{5} v_{i z}+d_{6}$

Data from experiment 1 were used to fit the coefficients of the polynomials from the measured $\mathbf{v}_{0}$ with respect to the launch plane and velocity at the exit of the launcher, $\mathbf{v}_{i}$. Notice that, since it was not possible to directly measure the $\mathbf{v}_{i}$ components with the motion tracking system due to the presence of the screen, their values were evaluated by means of the following relations:

$\left\{\begin{array}{l}v_{i x}=\mathrm{SP} \cos (\mathrm{EL}) \\ v_{i z}=\mathrm{SP} \sin (\mathrm{EL})\end{array}\right.$

with SP and EL representing the values set on the control software of the apparatus. Nevertheless motion inside the launcher could be altered by several random events, such as possible vibrations of the apparatus, as well as different contact regions and interactions between the ball and the delivery wheels due to variability of the position and velocity of the ball when inserted by the experimenter in the aperture between the delivery wheels. To estimate the random variability in the real SP and EL values, a further analysis was also carried out by means of Eqs. (17) and (18). Real EL and SP launch parameters were estimated starting from the observed $\mathbf{v}_{0}$ components, and the mean and standard deviation of the difference between estimated and set values were computed.

2.3.2.4. Estimation of $T$ and $Z$ variability as a function of launch parameters. The standard deviations of the flight parameters $\sigma_{T}$ and $\sigma_{Z}$ at distance $d$ from the launch plane, for any given couple of launch parameters (EL and SP), were also evaluated. A second degree polynomial representing the relationship between flight variance and initial conditions was fitted, estimating the coefficient of the following equations:

$$
\begin{aligned}
\sigma_{T}= & e_{1} \mathrm{SP}^{2}+e_{2} \mathrm{EL}^{2}+e_{3} d^{2}+e_{4} \mathrm{SPEL}+e_{5} \mathrm{SP} d+e_{6} \mathrm{EL} d \\
& +e_{7} \mathrm{SP}+e_{8} \mathrm{EL}+e_{9} d+e_{10}
\end{aligned}
$$

$$
\begin{aligned}
\sigma_{Z}= & f_{1} \mathrm{SP}^{2}+f_{2} \mathrm{EL}^{2}+f_{3} d^{2}+f_{4} \mathrm{SPEL}+f_{5} \mathrm{SP} d+f_{6} \mathrm{EL} d \\
& +f_{7} \mathrm{SP}+f_{8} \mathrm{EL}+f_{9} d+f_{10}
\end{aligned}
$$

2.3.2.5. Mapping the desired $T, Z$, and $d$ flight parameters into launch parameters. Once the polynomial coefficients were fitted as described above, the values of the launch parameters EL and SP to be set in the launching apparatus control software to obtain desired ball time flight $(T)$ and height $(Z)$ at a distance $d$ from the launch plane were computed as follows:

1. Given $T, Z, d$ values, Eqs. (12) and (13) were solved (Matlab, fsolve function with trust region dogleg method) in order to compute the corresponding $v_{0 x}$ and $v_{0 z}$ values.

2. Similarly, given the velocity components at the exit of the screen, $v_{0 x}$ and $v_{0 z}$, the launch parameters ( $\hat{S} P$ and $\hat{E} \mathrm{~L}$ ) were computed solving Eqs. (17) and (18) (Matlab, fsolve function).

3. Since the rotational speed of the disks of the Bola launching machine could be adjusted with a resolution in the exit speed of $1 \mathrm{mph}$, the closest integer value to ŜP was selected.

$T$ and $Z$ effective values due to launch speed resolution were then estimated using the forward mappings (Eqs. (17)-(19) and Eqs. (12) and (13)). The variability of $T$ and $Z, \hat{\sigma}_{T}, \hat{\sigma}_{Z}$, were also estimated (Eqs. (20) and (21)).

\subsection{Validation of the methodology}

Two tests were performed to evaluate the quality of the fit, the accuracy and the precision of the proposed methodology. The first test aimed at evaluating the quality of the polynomial fits with a cross-validation procedure. Data from experiment 1 were used in the analysis. In particular, $21 \mathrm{EL}_{\mathrm{v}}$ and $\mathrm{SP}_{\mathrm{v}}$ pairs, enclosed in the convex polygon obtained by scaling the convex hull of all 65 point in the EL-SP plane by $60 \%$ with respect to their center of mass (see Fig. 9, left panel), were selected. The polynomial fits described above were repeated 21 times, excluding each time one $\mathrm{EL}_{\mathrm{v}}$ and $\mathrm{SP}_{\mathrm{v}}$ pair. For each fit, the excluded launch parameters were used to estimate the ball flight parameters $(\hat{T}$ and $\hat{Z}$ ) at $d=6 \mathrm{~m}$ from the launcher (Eqs. (12), (13), (17) and (18)), and their variance (Eqs. (20) and (21)) and to compare those values with the measured values.

In the second test, a new experimental session (experiment 2 ) of launches was performed using a different ball from the one used in experiment 1 . From a set of desired ball flight parameters $(T=[0.50 .7] \mathrm{s}, Z=[1.31 .6] \mathrm{m}, d=6 \mathrm{~m})$ the launch parameter $(\mathrm{SP}, \mathrm{EL})$, to be set in the control panel of the launch apparatus, were computed following the procedure described above (Section 2.3.2.5) using the parameters of the polynomial functions fitted on the data of experiment 1 . For each combination of EL and SP values a block of 10 trials were performed and the position of the ball was recorded as previously described (Section 2.3.2.1). Desired and measured flight parameters $(T, Z)$ and their variance were compared and used to quantify the relative accuracy and precision of the method as follows, averaged over the different $T$ and $Z$ flight conditions:

Acc $=$ mean $\left(\frac{1-\left(\bar{V}-V_{\mathrm{d}}\right)}{V_{\mathrm{d}}}\right)$,

Prec $=\operatorname{mean}(1-\operatorname{mean}(\mathrm{CV}))$

where CV is the coefficient of variation computed as $\mathrm{CV}=(\sigma / \bar{V})$, $V_{\mathrm{d}}$ is the desired value, $\bar{V}$ is the sample mean of the measured distribution, and $\sigma$ is its standard deviation. Both $V_{\mathrm{d}}, \bar{V}$, and $\sigma$ were different for each EL and SP combination.

\section{Results}

\subsection{Effect of air drag on ball trajectory}

Fig. 5 shows the comparison between the paths (in a vertical plane) of real balls, tracked with a motion capture system, and the ideal paths extrapolated from the measured initial velocity neglecting air drag. The aerodynamic forces acting on the projectile make the real ball paths (solid lines) deviate from the paths extrapolated assuming no drag (dashed lines). In the example, after a flight duration of $0.6 \mathrm{~s}$ the real ball is located $0.48 \pm 0.11 \mathrm{~m}$ below the exit hole and $6.03 \pm 0.02 \mathrm{~m}$ from to the screen, while for the same initial velocity the ideal ball is $0.43 \pm 0.10 \mathrm{~m}$ below the exit hole and $7.34 \pm 0.04 \mathrm{~m}$ from the screen. Differences between real and ideal ball locations at $T=0.6 \mathrm{~s}$ were highly significant for both $\mathrm{x}$ and $y$ coordinates $(X: t(9)=76.74, p<0.001 ; Z: t(9)=16.3, p<0.001)$. Thus, in many experimental conditions air drag cannot be neglected without introducing large errors. 


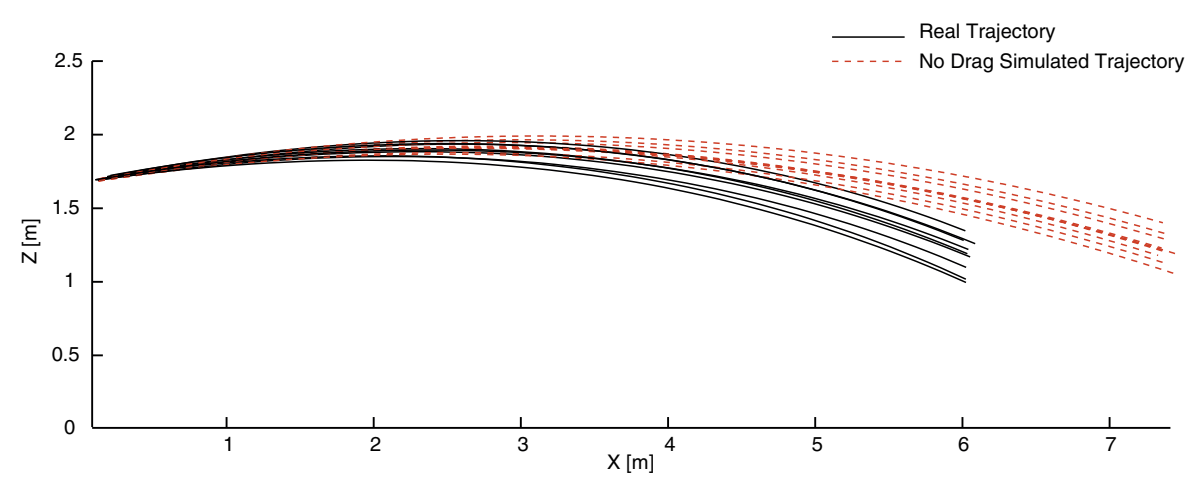

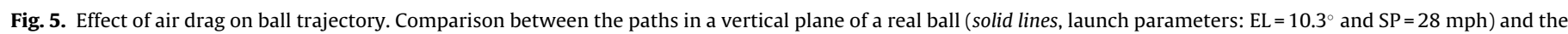

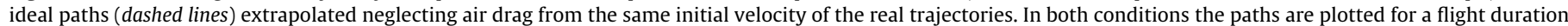
of $600 \mathrm{~ms}$.

\subsection{Position of the ball in the exit hole}

With respect to the position of the center of the exit hole, the $y$ and $z$ coordinates of the center of the ball when it crossed the screen plane were $\Delta y_{0}=0.2 \pm 0.6 \mathrm{~cm}$ (mean \pm st. dev., range [ $\left.-2.81 .7\right]$ ) and $\Delta z_{0}=3.5 \pm 0.6 \mathrm{~cm}$ (range $[0.35 .2] \mathrm{cm}$ ). Thus, the ball tended to pass through the screen slightly above the hole center but the distributions of the $y$ and $z$ coordinates (Fig. 6) had small variances around the grand means. Such results support the assumptions adopted in Section 2.3.1 for the definition of $Z_{\text {launcher }}$ as a function only of the launcher elevation (EL). All in all, the apparatus guarantees the desired standardization of the location of the visual stimuli at launch time.

\subsection{Calibration}

Results of the system calibration at a distance of $6 \mathrm{~m}$ from the launcher are shown in Figs. 7 and 8. Values of the coefficients of the polynomial fits of the flight duration $(T)$ and ball height $(Z)$ as a function of the initial velocity $\left(v_{0 x}, v_{0 z}\right)$, of the initial velocity as a function of ball release speed (SP) and elevation (EL), and of flight duration and ball height standard deviations as a func-
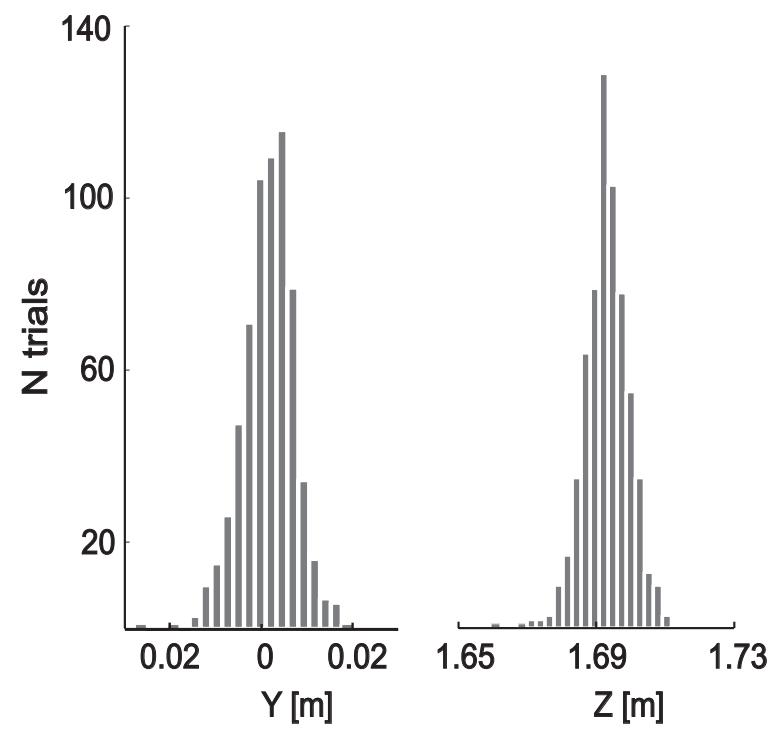

Fig. 6. Position of the ball in the exit hole. The distributions of the lateral $(y)$ and vertical $(z)$ coordinates of the ball position on the launch plane for all 660 launches (experiment 1 , see Section 2 ) had a small standard deviation $(0.6 \mathrm{~cm}$ for both coordinates), thus the position of the ball is standardized for different launch parameters. tion of the launch parameters are given in Table 2 . The polynomial coefficients are derived from flight parameters measured in a large number of trajectories launched with different launch parameters (experiment 1, see Section 2.3.2). Once fitted, the polynomial coefficients can be used to estimate the velocity vector components $\left(v_{0 x}, v_{0 z}\right)$ at the exit hole from the SP and EL values (Fig. 7B), and subsequently, the ball flight parameters $T$ and $Z$ at distance $d$ (see Fig. 8A). The expected variability of the flight parameters (Fig. 8B) showed that a ball could be delivered with a flight time standard deviation of at most of $20 \mathrm{~ms}$ from the desired value, while standard deviation in $Z$ could arrive in a few cases to $40 \mathrm{~cm}$ ( 5 over 65 SP and EL combinations), but remained in $97 \%$ of cases below $30 \mathrm{~cm}$ and in the $80 \%$ of the cases below $20 \mathrm{~cm}$. The high $R^{2}$ values of the fits indicate that the chosen polynomials very accurately model the relationship between launch and flight parameters. However, quality of the fit of the relationship between initial launch parameters (EL and SP) and $v_{0 z}$ velocity component was lower with respect to the others $\left(R^{2}=0.9\right)$. In this respect, the analysis on the estimated EL and SP launch parameters values, computed from the measured $v_{0}$ components (see Section 2.3.2.3), showed average errors of $0.00 \pm 0.33 \mathrm{mph}$ for SP and $0.24 \pm 1.12 \mathrm{deg}$ for EL, with $R^{2}$ values of 0.99 and 0.92 , respectively. Thus the lower quality of the reconstruction of $v_{0 z}$ can be attributed mainly to the higher variability of the effective launch EL.

\subsection{Validation}

We first assessed the error in the estimation of the ball flight duration $(T)$ and height $(Z)$ at a distance $(d)$ of $6 \mathrm{~m}$ from the launcher with a cross-validation procedure. Polynomial parameters were fitted excluding each time one of 21 sets of launch parameters (SP and EL) selected in the central region of the parameter space (Fig. 9), from the 65 pairs of experiment 1. Analysis of the difference between measured and predicted flight parameters revealed that the root mean squared error (RMSE) in the estimation flight duration $(T)$ and ball height $(Z)$ was, respectively, $2.72 \mathrm{~ms}$ and $5.48 \mathrm{~cm}$. Indeed, the linear regressions between predicted and the observed ball flight parameters were significant, with a slope of 0.994 for $T\left(F(1,19)=3.3 \cdot 10^{4}, p<0.001\right)$ and 1.004 for $Z\left(F(1,19)=1.3 \cdot 10^{3}\right.$, $p<0.001)$. A $t$-statistic test revealed that both slopes were not statistically different from $1(T: t(19)=-1.099, p=0.14 ; Z: t(19)=0.155$, $p=0.56$ ). The higher variance of $Z$ with respect to $T$, can be attributed to the higher variability in $v_{0 z}$ components with respect to $v_{0 x}$ reported above, and related to occasional external causes affecting EL launch parameter described in Section 2.3.2.3.

A second validation test was performed to assess the accuracy and precision of apparatus in generating desired flight parameters. The comparison between desired (dashed horizontal lines), 
T

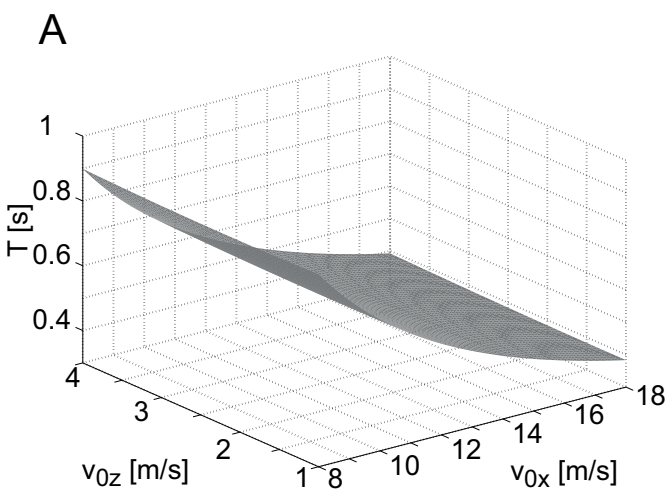

$\mathrm{v}_{0 \mathrm{x}}$

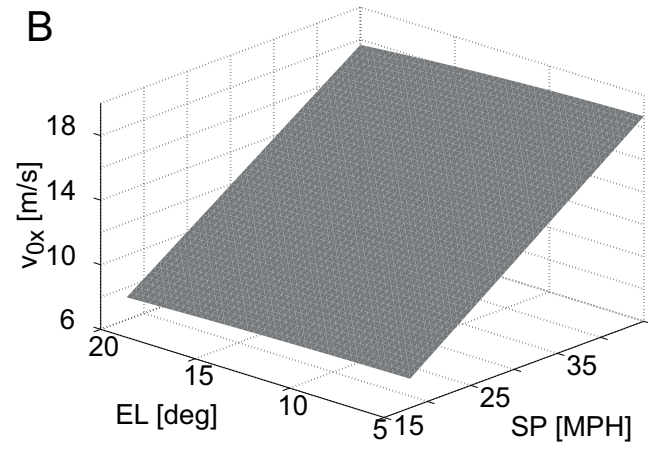

Z

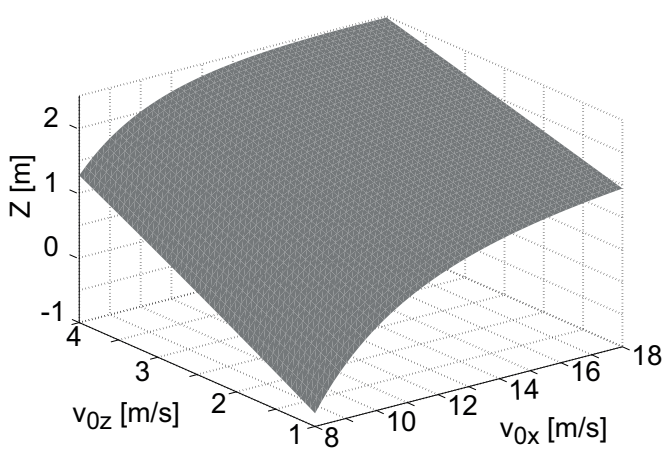

$\mathrm{v}_{0 z}$

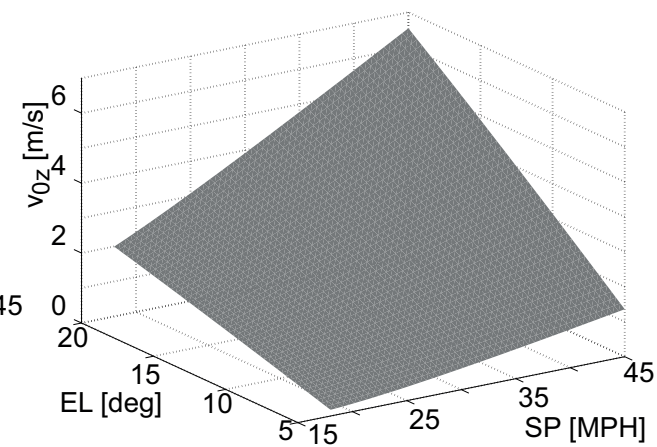

Fig. 7. Relationships between launch parameters, initial ball velocity, and flight parameters approximated by polynomial functions. Coefficients of the polynomials are fitted on a large dataset of ball flight trajectories (experiment 1). (A) Relationship between velocity components $\left(v_{0 x}, v_{0 z}\right)$ and flight duration $(T)$ and ball height $(Z)$ at a distance of $6 \mathrm{~m}$ from the launch plane. (B) Relationship between launch parameters and velocity components on the launch plane.
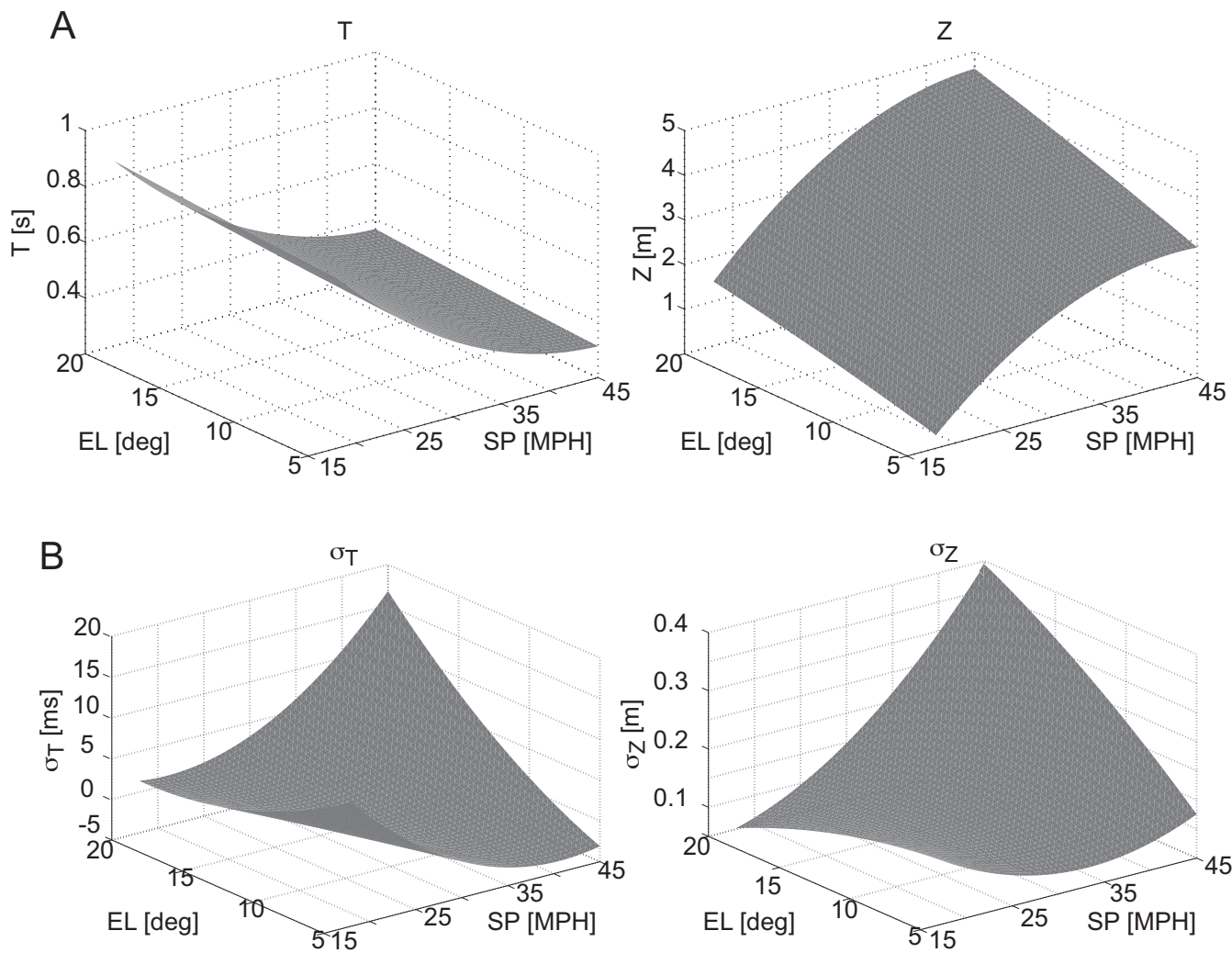

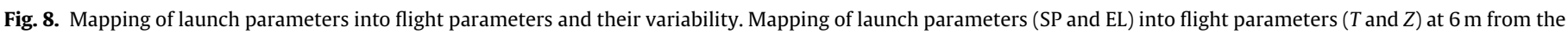
launch plane $(A)$, obtained combining the two mappings of Fig. 7, and standard deviations of the flight parameters estimated by a third polynomial fit (B). 
Table 2

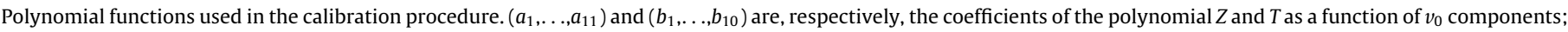

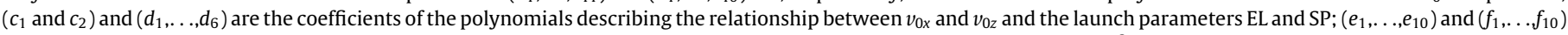
are used to estimate the variance of the $T$ and $Z, \sigma_{T}$ and $\sigma_{Z}$, for any given EL and SP combination. The last row reports the $R^{2}$ values of the fits.

\begin{tabular}{|c|c|c|c|c|c|c|c|c|c|c|c|}
\hline \multicolumn{2}{|c|}{$Z\left(v_{0 x}, v_{0 z}\right)$} & \multicolumn{2}{|c|}{$T\left(v_{0 x}, v_{0 z}\right)$} & \multicolumn{2}{|c|}{$v_{0 x}(\mathrm{EL}, \mathrm{SP})$} & \multicolumn{2}{|c|}{$v_{0 z}(\mathrm{EL}, \mathrm{SP})$} & \multicolumn{2}{|c|}{$\sigma_{T}(\mathrm{EL}, \mathrm{SP})$} & \multicolumn{2}{|c|}{$\sigma_{Z}(\mathrm{EL}, \mathrm{SP})$} \\
\hline$a_{1}$ & 1.37 & $b_{1}$ & $2.97 \cdot 10^{-3}$ & $c_{1}$ & 0.405 & $d_{1}$ & 0.503 & $e_{1}$ & $2.70 \cdot 10^{-5}$ & $f_{1}$ & $3.08 \cdot 10^{-4}$ \\
\hline$a_{2}$ & 1.54 & $b_{2}$ & $2.27 \cdot 10^{-2}$ & $c_{2}$ & 0.537 & $d_{2}$ & $1.61 \cdot 10^{-2}$ & $e_{2}$ & $4.05 \cdot 10^{-5}$ & $f_{2}$ & $-3.24 \cdot 10^{-4}$ \\
\hline$a_{3}$ & -0.78 & $b_{3}$ & $4.58 \cdot 10^{-2}$ & & & $d_{3}$ & -0.01 & $e_{3}$ & $1.68 \cdot 10^{-4}$ & $f_{3}$ & $5.60 \cdot 10^{-4}$ \\
\hline$a_{4}$ & -9.56 & $b_{4}$ & $3.94 \cdot 10^{-3}$ & & & $d_{4}$ & -1.47 & $e_{4}$ & $7.08 \cdot 10^{-5}$ & $f_{4}$ & $9.15 \cdot 10^{-4}$ \\
\hline$a_{5}$ & -1.47 & $b_{5}$ & $-4.11 \cdot 10^{-3}$ & & & $d_{5}$ & -1.76 & $e_{5}$ & $4.71 \cdot 10^{-5}$ & $f_{5}$ & $1.21 \cdot 10^{-3}$ \\
\hline$a_{6}$ & -0.34 & $b_{6}$ & $1.12 \cdot 10^{-2}$ & & & $d_{6}$ & 13.0 & $e_{6}$ & $1.42 \cdot 10^{-4}$ & $f_{6}$ & $1.07 \cdot 10^{-3}$ \\
\hline$a_{7}$ & -5.26 & $b_{7}$ & $-8.16 \cdot 10^{-2}$ & & & & & $e_{7}$ & $-2.83 \cdot 10^{-3}$ & $f_{7}$ & $-3.22 \cdot 10^{-2}$ \\
\hline$a_{8}$ & 7.06 & $b_{8}$ & $-1.98 \cdot 10^{-2}$ & & & & & $e_{8}$ & $-3.73 \cdot 10^{-3}$ & $f_{8}$ & $-2.15 \cdot 10^{-2}$ \\
\hline$a_{19}$ & 25.4 & $b_{9}$ & $8.71 \cdot 10^{-2}$ & & & & & $e_{9}$ & $-3.86 \cdot 10^{-3}$ & $f_{9}$ & $-2.43 \cdot 10^{-2}$ \\
\hline$a_{10}$ & 40.5 & $b_{10}$ & 1.01 & & & & & $e_{10}$ & $7.65 \cdot 10^{-2}$ & $f_{10}$ & $6.47 \cdot 10^{-1}$ \\
\hline$a_{11}$ & -136 & & & & & & & & & & \\
\hline$R^{2}$ & 0.985 & $R^{2}$ & 0.999 & $R^{2}$ & 0.998 & $R^{2}$ & 0.905 & $R^{2}$ & 0.999 & $R^{2}$ & 0.989 \\
\hline
\end{tabular}

measured (black markers and standard deviation error bars), and estimated flight parameters $T$ (top) and $Z$ (bottom) at $d=6 \mathrm{~m}$ for data collected in a separate validation experiment, using a different ball, with 4 pairs of flight parameters is illustrated in Fig. 10. Relative accuracy was $98.8 \%$ for $T\left(T_{1}=98.7 \%, T_{2}=98.9 \%\right)$, and $96.6 \%$ for $Z\left(Z_{1}=98.3 \%, Z_{2}=95 \%\right)$; relative precision $98.4 \%$ for $T\left(T_{1}=98.7 \%\right.$, $\left.T_{2}=98.2 \%\right)$ and $97.4 \%$ for $Z\left(Z_{1}=97.2 \%, Z_{2}=97.5 \%\right)$. The mean error between measured and estimated values was $22 \mathrm{~ms}$ for $T$ and $4.04 \mathrm{~cm}$ for $Z$.

In summary, these results indicate that the designed apparatus can deliver balls with desired spatial and temporal flight parameters with high precision and accuracy.

\section{Discussion}

We have developed and validated a novel ball launching system that can be used to explore the motor control strategies involved in catching, hitting or, more generally, interceptive actions on flying balls. The system is able to deliver a ball at a given distance from the launcher controlling its spatial position and flight duration taking into account air drag. Ball motion is tracked throughout the flight by means of a motion capture system. We showed that the effect of air drag on ball flight is not negligible in a typical ball catching experiment (Fig. 5). Our system is based on a commercial ball launching machine used to train cricket players, mounted on an actuated structure which could be opportunely displaced and rotated to properly adjust the initial orientation and speed of the projectile trajectory. Furthermore, prior vision of the ball inside the launcher at release was blocked by forcing the ball to pass through a hole in a screen hiding the entire apparatus. Finally, regardless of the required initial trajectory parameters, the system guarantees a standard position of the ball at launch time.

Careful calibration methodology was developed in order to determine, based on the desired delivery trajectory $T-Z$ characteristics, the ball initial flight parameters expressed in terms of vertical inclination (EL) and initial velocity (SP), to be set on the control panel of the apparatus. Overall relative accuracy and precision of the system in the prediction of ball flight characteristics evaluated by means of dedicated validation tests were $>96 \%$, with average error, respectively, of $4.04 \mathrm{~cm}$ for the $Z$ variable and $22 \mathrm{~ms}$ for $T$ variable. These results guaranteed setting the SP and EL values within the ranges used during the calibration phase, which must be chosen appropriately depending on the desired range of experimental conditions. To our knowledge, no previous study dealing with catching of flying balls has employed a launching apparatus able to vary the experimental conditions automatically and to control ball flight characteristics systematically. In previous studies, the initial height of the ball and the launch angle has been set manually and regulated empirically to obtain desired ball flight parameters. In some cases, in order to quantify variability and accuracy of launches, landing or delivery location of balls projected with different launcher configurations and speeds were recorded. With this approach, Tijtgat et al. (2009) obtained coefficients of variation for ball height at delivery ranging from $0.9 \%$ to $3.2 \%$, which are similar to our CV values ranging between $2 \%$ and $3 \%$ for ball height, and between $0.9 \%$ and $2.4 \%$ for flight time. Some investigators have quantified ball delivery accuracy within a circular area of approximately $0.3 \mathrm{~m}^{2}$ (Button et al., 2000, 2002). Other investigators have reported a range of landing distances from the intended one of $\pm 1 \mathrm{~m}$ (Oudejans et al., 1997). Finally, others (Laurent et al., 1994; Mazyn et al., 2006, 2007) did not perform any preliminary calibration test, but accepted only
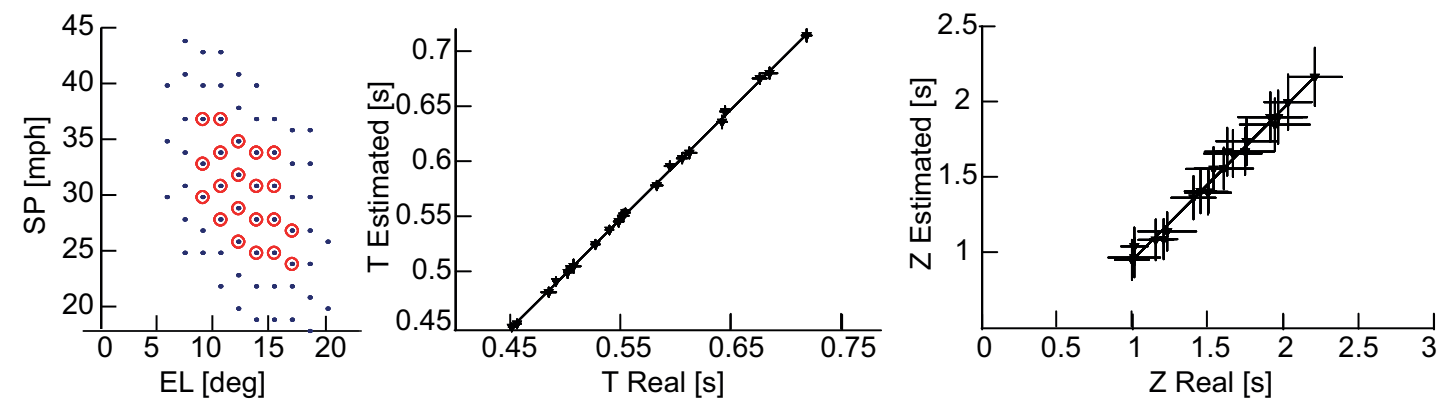

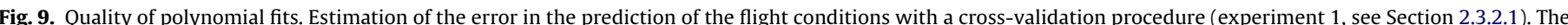

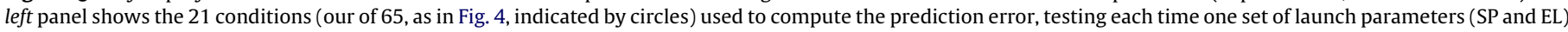

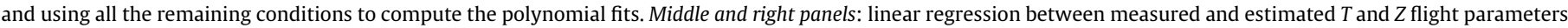
evaluated at $d=6 \mathrm{~m}$ from the launch plane. 

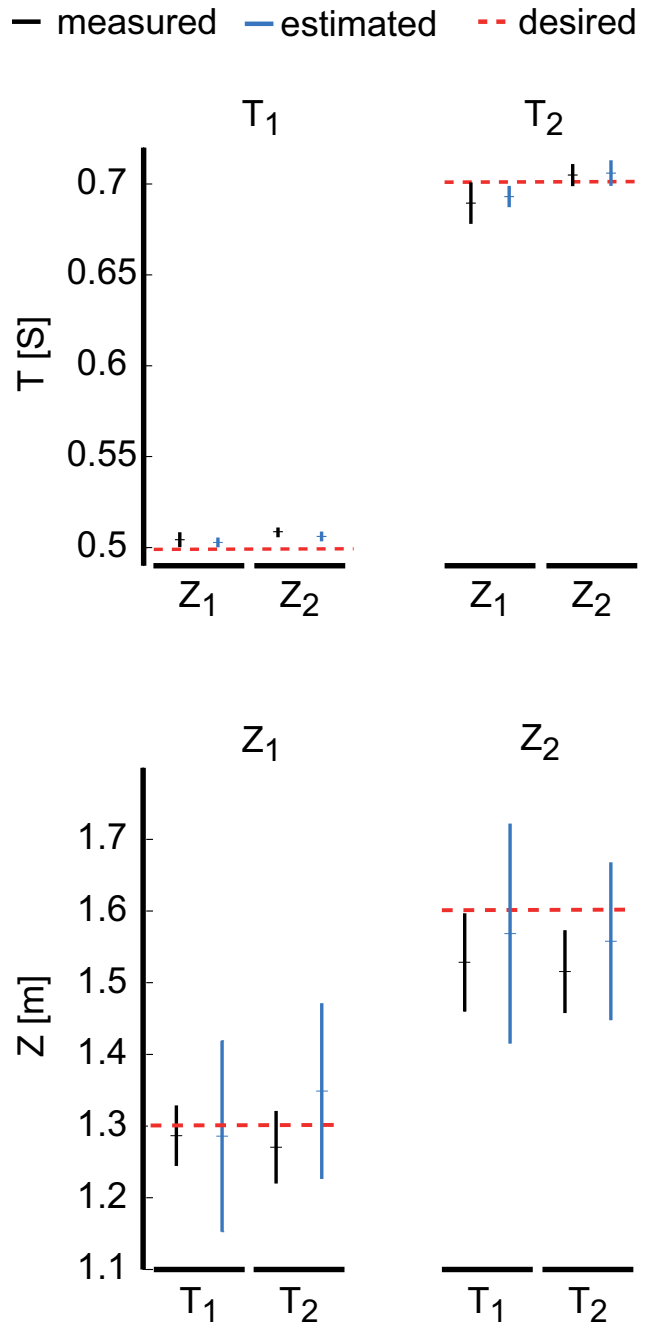

Fig. 10. Accuracy and precision of launching system. The differences between desired, estimated, and measured $T$ and $Z$ flight parameters (at $d=6 \mathrm{~m}$ distance from the launch plane) were evaluated in a second experimental session (experiment 2 ) different from the one used to fit the polynomial functions (experiment 1). Given desired $T$ and $Z$ values (red dashed lines), polynomial functions were used to compute the SP and EL that were set in the launching system. Ball trajectory tracking allowed to measure the mean and standard deviation of the actual $T$ and $Z$ (black lines) for each of the 4 sets of launch conditions. Finally, the same polynomial functions were used to compute an estimate of the mean and standard deviation for $T$ and $Z$ (gray lines). (For interpretation of the references to color in this figure legend, the reader is referred to the web version of the article.)

balls reaching the target in an imaginary circle of $30 \mathrm{~cm}$ in diameter with its center at a predefined height (i.e. $15 \mathrm{~cm}$ above shoulder of the catching arm). Our results from the estimation of the $T$ and $Z$ variability reported in Fig. 8B, show that balls could be delivered with a standard deviation in $Z$ less than $30 \mathrm{~cm}$ in most of the cases (97\%).

Most of the experiments dealing with catching tasks involved the adjustment of ball height at delivery depending on a particular body reference point (i.e. shoulder or knee height). In these contexts, launch parameters and launcher configuration were adjusted differently for each participant depending on the required conditions at impact. As a consequence, initial ball position and visual stimuli could change among trials and experimental sessions (Button et al., 2000, 2002; Laurent et al., 1994; Mazyn et al., 2006, 2007; Tijtgat et al., 2009). In a few cases of such previous studies, the launching angle was maintained in order to keep the flight trajectory of the ball identical from the viewpoint of the participant, thus, lifting up or down the entire device to achieve the required launch condition (Mazyn et al., 2007; Oudejans et al., 1997). Initial ball position as well as launcher orientation can be used by catchers to estimate the ball trajectory prior to launch, a potential confounding factor in a catching experiment. Indeed, during the experiment a modification of the launcher configuration, corresponding to a new experimental condition, may provide information on the resulting change in the ball trajectory characteristics to the catcher. For example, a higher elevation angle may pre-cue the catcher of a ball trajectory with a higher impact height and a longer flight time. Similarly, at a given orientation, a higher position of the launcher may suggest a higher impact point. Expert catchers are more effective in using early information about initial ball trajectory characteristics (Land and McLeod, 2000), but the capacity to anticipate the ball's path and successfully accomplish the task could be present also in naïve subjects. Thus, besides the accuracy in prediction, or the enrollment of skilled instead of inexpert catchers, prior knowledge of initial conditions could affect motor responses both in terms of timing and strategy. In this context, the launching apparatus developed in the present work, by impeding prior vision of launcher orientation and providing always the same release position at launch, represents a significant methodological advancement because it allows for more reliable comparisons across subjects useful for the identification of general motor control strategies. Finally, auditory cues, such as the noise generated by the interaction of the ball with the delivery wheels, might also provide information about the timing of the launch before the ball exit the hole on the screen and becomes visible. To prevent such auditory anticipation, it may be sufficient to mask any ball delivery noise using earphones.

The calibration methodology developed in this study aimed at both systematically and automatically actuating the launch apparatus and, taking in to account air resistance, controlling the ball flight parameters. Analysis of the polynomial fitting residuals (see Table 2) showed high $R$-square values larger than 0.99 for all fits except the one that predicted the $v_{0 z}$ components from the EL and SP values $\left(R^{2}=0.90\right)$. This discrepancy is likely related to the possible random factors affecting the ball motion at release. Indeed, when using a launching machine, the interaction between the ball and the apparatus could be affected by the insert action of a particular experimenter who applies an initial velocity to the ball altering the pushing action of the disks. This interference can be accidental, hence an off line analysis would provide evidence for atypical ball motion behavior with respect to other launches, but it could also be systematic, depending on the particular way the experimenter interacts with the machine. Also, during flight, possible non-homogeneous ball surfaces, due for example to the presence of dimples or overlapped edges of the reflecting tape, can produce lift effects reducing air drag action during the flight and therefore increasing or reducing the carry through the air. Such effects were visible in Fig. 5: the same initial set values of ball velocity and trajectory inclination (EL and SP) could return different ball characteristics at delivery. Variability in the $Z$ variable cannot be completely eliminated, but can be quantified by tracking ball position. Indeed, if adequately monitored and restrained to a range (i.e. $\pm 30 \mathrm{~cm}$, as in Mazyn et al., 2006, 2007), variability can be exploited to explore the ability of the CNS to overcome and interpret small fluctuations requiring an on line control of the external stimuli, thus avoiding automatic motor responses as well as anticipation.

In conclusion, the new launching system that we designed, calibrated and tested provides a novel and robust instrument to be used in catching experiments in which a high reliability and accuracy of ball trajectory in space parameters is required. Such system will automatically allow to control over the orientation of the launcher as well as the ball release speed, to track the ball motion throughout the flight, and to control experimental conditions systematically, in naturalistic interceptive tasks in three-dimensional space. 


\section{Acknowledgments}

Supported by the Italian Ministry of Health, the Italian Space Agency (DCMC and CRUSOE), and the EU Seventh Framework Programme (FP7-ICT No 248311 AMARSi and No 247959 MINDWALKER). The authors are grateful to Michael J. MacLellan for helpful comments to improve the readability of the paper.

\section{References}

Bennett S, van der Kamp J, Savelsbergh GJ, Davids K. Timing a one-handed catch. I. Effects of telestereoscopic viewing. Exp Brain Res 1999;129:362-8.

Bockemuhl T, Troje NF, Durr V. Inter-joint coupling and joint angle synergies of human catching movements. Hum Mov Sci 2009;29:73-93.

Brenner E, Smeets JB. Flexibility in intercepting moving objects. J Vision 2007;7(14):1-7.

Brouwer AM, Brenner E, Smeets JB. Hitting moving objects. The dependency of hand velocity on the speed of the target. Exp Brain Res 2000;133:242-8.

Brouwer AM, Brenner E, Smeets JB. Hitting moving objects: is target speed used in guiding the hand? Exp Brain Res 2002a;143:198-211.

Brouwer AM, Brenner E, Smeets JB. Perception of acceleration with short presentation times: can acceleration be used in interception? Percept Psychophys 2002b;64:1160-8.

Brouwer AM, Middelburg T, Smeets JB, Brenner E. Hitting moving targets: a dissociation between the use of the target's speed and direction of motion. Exp Brain Res 2003:152:368-75.

Button C, Davids K, Bennett SJ, Savelsbergh GJ. Anticipatory responses to perturbation of co-ordination in one-handed catching. Acta Psychol (Amst) 2002;109:75-93.

Button C, Davids K, Bennett SJ, Taylor MA. Mechanical perturbation of the wrist during one-handed catching. Acta Psychol (Amst) 2000;105:9-30.

De Mestre N. The mathematics of projectiles in sprt. Cambridge: Cambridge University Press; 1990.

Dessing JC, Wijdenes LO, Peper CL, Beek PJ. Adaptations of lateral hand movements to early and late visual occlusion in catching. Exp Brain Res 2009;192:669-82.

Lacquaniti F, Carrozzo M, Borghese NA. Time-varying mechanical behavior of multijointed arm in man. J Neurophysiol 1993;69:1443-64.

Lacquaniti F, Maioli C. Adaptation to suppression of visual information during catching. J Neurosci 1989;9:149-59.

Lacquaniti F, Maioli C. Anticipatory and reflex coactivation of antagonist muscles in catching. Brain Res 1987;406:373-8.

Land MF, McLeod P. From eye movements to actions: how batsmen hit the ball. Nat Neurosci 2000;3:1340-5.

Laurent M, Montagne G, Savelsbergh GJ. The control and coordination of one-handed catching: the effect of temporal constraints. Exp Brain Res 1994;101:314-22.

Lee DN. The optic flow field: the foundation of vision. Philos Trans R Soc Lond B: Biol Sci 1980;290:169-79.

Lopez-Moliner J, Brenner E, Smeets JB. Effects of texture and shape on perceived time to passage: knowing "what" influences judging "when". Percept Psychophys 2007a;69:887-94.

Lopez-Moliner J, Field DT, Wann JP. Interceptive timing: prior knowledge matters. J Vision 2007b;7(11):1-8.

Mazyn LI, Montagne G, Savelsbergh GJ, Lenoir M. Reorganization of catching coordination under varying temporal constraints. Motor Control 2006;10:143-59.
Mazyn LI, Savelsbergh GJ, Montagne G, Lenoir M. Planning and on-line control of catching as a function of perceptual-motor constraints. Acta Psychol (Amst) 2007;126:59-78.

McBeath MK, Shaffer DM, Kaiser MK. How baseball outfielders determine where to run to catch fly balls. Science 1995;268:569-73.

McLeod P, Dlenes Z. Running to catch the ball. Nature 1993;362:23.

McLeod P, Reed N, Dienes Z. Toward a unified fielder theory: what we do not yet know about how people run to catch a ball. J Exp Psychol Hum Percept Perform 2001;27:1347-55.

Montagne G, Laurent M, Durey A, Bootsma R. Movement reversals in ball catching. Exp Brain Res 1999;129:87-92.

Mrotek LA, Soechting JF. Target interception: hand-eye coordination and strategies. J Neurosci 2007;27:7297-309.

Oudejans RR, Michaels CF, Bakker FC. The effects of baseball experience on movement initiation in catching fly balls. J Sports Sci 1997;15(6):587-95.

Peper L, Bootsma RJ, Mestre DR, Bakker FC. Catching balls: how to get the hand to the right place at the right time. J Exp Psychol Hum Percept Perform 1994;20:591-612.

Regan D. Visual factors in hitting and catching. J Sports Sci 1997;15:533-58.

Regan D, Beverley K, Cynader M. The visual perception of motion in depth. Sci Am 1979;241:136-51.

Regan D, Beverley KI. Looming detectors in the human visual pathway. Vision Res 1978;18:415-21.

Savelsbergh G, Bennett S, van der Kamp J. Interceptive action in sport. London: Routledge: 2002.

Senot P, Zago M, Lacquaniti F, McIntyre J. Anticipating the effects of gravity when intercepting moving objects: differentiating up and down based on nonvisual cues. J Neurophysiol 2005;94:4471-80.

Shepard R. Perceptual and cognitive universals as reflections of the world. Psychon Bull Rev 1994;1:2-28.

Tijtgat P, Bennett SJ, Savelsbergh GJ, De Clercq D, Lenoir M. Advance knowledge effects on kinematics of one-handed catching. Exp Brain Res 2009;201:875-84.

Tresilian JR. Analysis of recent empirical challenges to an account of interceptive timing. Percept Psychophys 1999a;61:515-28.

Tresilian JR. Visually timed action: time-out for 'tau'? Trends Cogn Sci 1999b;3:301-10.

Tresilian JR, Lonergan A. Intercepting a moving target: effects of temporal precision constraints and movement amplitude. Exp Brain Res 2002;142:193-207.

Tresilian JR, Plooy A. Systematic changes in the duration and precision of interception in response to variation of amplitude and effector size. Exp Brain Res 2006;171:421-35.

Tresilian JR, Plooy AM, Marinovic W. Manual interception of moving targets in two dimensions: performance and space-time accuracy. Brain Res 2009;1250:202-17.

van der KampJ, Savelsbergh G, Smeets J. Multiple information sources in interceptive timing. Hum Movement Sci 1997;16:787-821.

Wann JP. Anticipating arrival: is the tau margin a specious theory? J Exp Psychol Hum Percept Perform 1996;22:1031-48.

Zago M, Bosco G, Maffei V, Iosa M, Ivanenko YP, Lacquaniti F. Fast adaptation of the internal model of gravity for manual interceptions: evidence for eventdependent learning. J Neurophysiol 2005;93:1055-68.

Zago M, Lacquaniti F. Cognitive, perceptual and action-oriented representations of falling objects. Neuropsychologia 2005;43:178-88.

Zago M, McIntyre J, Senot P, Lacquaniti F. Visuo-motor coordination and internal models for object interception. Exp Brain Res 2009;192:571-604. 